\title{
Assessing an Impulsive Model for Rotational Energy Partitioning to Acetyl Radicals from the Photodissociation of Acetyl Chloride at 235 nm
}

\author{
Caroline C. Womack, ${ }^{\dagger}$ Wei-Hai Fang, ${ }^{\dagger}$ Daniel B. Straus, ${ }^{\dagger}$ and Laurie J. Butler $*, \dagger$ \\ The James Franck Institute and the Department of Chemistry, The University of Chicago, Chicago, \\ Illinois 60637, United States, and College of Chemistry, Beijing Normal University, \\ Beijing 100875, People's Republic of China
}

Received: August 10, 2010; Revised Manuscript Received: November 8, 2010

\begin{abstract}
This work uses the photodissociation of acetyl chloride to assess the utility of a recently proposed impulsive model when the dissociation occurs on an excited electronic state that is not repulsive in the Franck-Condon region. The impulsive model explicitly includes an average over the vibrational quantum states of acetyl chloride when it calculates an impact parameter for fission of the $\mathrm{C}-\mathrm{Cl}$ bond, as well as the distribution of thermal energy in the photolytic precursor. The experimentally determined stability of the resulting acetyl radical to subsequent dissociation is the key observable that allows us to test the model's ability to predict the partitioning of energy between rotation and vibration of the radical. We compare the model's predictions for three different assumed geometries at which the impulsive force might act, generating the relative kinetic energy and the concomitant rotational energy in the acetyl radical. Assuming that the impulsive force acts at the transition state for $\mathrm{C}-\mathrm{Cl}$ fission on the $\mathrm{S}_{1}$ excited state gives a poor prediction; the model predicts far more energy in rotation of the acetyl radical than is consistent with the measured velocity map imaging spectrum of the stable radicals. The best prediction results from using a geometry derived from the classical trajectory calculations on the excited state potential energy surface. We discuss the insight gained into the excited state dissociation dynamics of acetyl chloride and, more generally, the utility of using the impulsive model in conjunction with excited state trajectory calculations to predict the partitioning of internal energy between rotation and vibration for radicals produced from the photolysis of halogenated precursors.
\end{abstract}

\section{Introduction}

In both molecular beam and bulk kinetics experiments, the photodissociation of halogenated precursors is often used to generate radical intermediates under controlled conditions in order to study the elementary chemical reactions of these highly reactive species. The reactions of photolytically produced radicals are influenced by how the energy initially imparted to these radicals is partitioned between translational, vibrational, and rotational energy. To understand this energy partitioning, a wide variety of models have been developed and tested in the last few decades. This paper seeks to assess a recently proposed impulsive model ${ }^{1}$ for its value in predicting the energy partitioning when the photolytic precursor is excited to an electronic state that is not repulsive in the vertical geometry, but rather has a $\mathrm{C}$-halogen bond fission transition state outside the Franck-Condon region.

Statistical, ${ }^{2}$ Franck-Condon,,${ }^{3,4}$ and direct impulsive models ${ }^{5}$ have been used to predict how the available energy in a unimolecular or bimolecular reaction is distributed between relative kinetic energy of the products and the internal vibrational and rotational energy of the products. While a statistical, or phase space, prediction for the energy partitioning to the product degrees of freedom is useful for many systems, if the dissociation dynamics are on a repulsive excited state or if there are significant inter- or intramolecular forces beyond the transition state, statistical predictions can be quite poor. A Franck-Condon approach ${ }^{3,4}$ can be useful to predict excitation

\footnotetext{
* Corresponding author. E-mail: L-Butler@uchicago.edu.

${ }^{\dagger}$ The University of Chicago.

* Beijing Normal University.
}

in product vibrational degrees of freedom. For photodissociation on steeply repulsive excited surfaces, an impulsive model ${ }^{5,6}$ for energy partitioning to translational and rotational energy is more appropriate than a statistical prediction. The early pioneering studies by Wilson and co-workers ${ }^{5-7}$ outlined two limiting cases $^{5,6}$ for such a photodissociation. Their rigid radical impulsive model does not allow for the partitioning of energy to product vibration but offers a prediction for the partitioning of the available energy between rotation and relative kinetic energy of the photofragments. Their soft radical limit allows for partitioning to molecular vibration as well. The barrier impulsive model modification introduced by North et al. ${ }^{8}$ for acetone photodissociation separately treats the energy released from dissociating down an exit barrier as impulsively partitioned, and the rest of the available energy as statistically partitioned. Though a few systems are well predicted by the original limiting cases of impulsive dissociation, ${ }^{9}$ the largely arbitrary assumptions in methods attempting to mix impulsive models with vibrational excitation ${ }^{4}$ calculated from Franck-Condon overlap with product states is still best described by Busch and Wilson's early comment: "...it must be admitted that a direct model based on a combination of intra- and interfragment effects is so spineless that it can match almost any photofragment spectrum." ${ }^{\prime 6}$ While exact quantum dynamics simulations on a global potential energy surface can reasonably predict the energy partitioning in systems with just a few atoms, such calculations are not practical for larger polyatomics. Thus, improvements in the simple models are needed to obtain reasonable estimates of the energy partitioning to photolytically produced polyatomic radicals. 
To gain quantitative predictive ability for energy partitioning in the photodissociation of halogenated polyatomics used as radical precursors, we have recently introduced an impulsive model $^{1}$ that has three key features. It (1) retains the essential component of classical conservation of angular momentum to predict the resulting radical's rotational energy but uses the measured recoil kinetic energy distribution rather than making a soft or rigid radical approximation, ${ }^{10}(2)$ explicitly includes a distribution of predicted impact parameters over the quantum mechanical vibrational wave function of the photolytic precursor, ${ }^{1}$ and (3) explicitly includes the distribution of vibrational energy in the photolytic precursor. ${ }^{1}$ When applied to the highly internally excited $\mathrm{CH}_{2} \mathrm{CH}_{2} \mathrm{OH}$ radicals formed from the photodissociation of 2-bromoethanol at $193 \mathrm{~nm}$, this model accurately predicted the percentage of radicals that were partitioned enough rotational energy at each relative kinetic energy to remain stable to further dissociation. ${ }^{1}$ The excited state of 2-bromoethanol is known to be repulsive in the Franck-Condon region, ${ }^{11,12}$ which made an impulsive model a reasonable assumption. In this paper, we test the utility of the model for predicting the partitioning of internal energy between rotation and vibration for the dissociation of a molecule, acetyl chloride, not on a steeply repulsive potential, but on an excited state with a $\mathrm{C}$-halogen bond fission transition state outside the FranckCondon region.

The photodissociation of acetyl chloride to the acetyl radical $\left(\mathrm{CH}_{3} \mathrm{CO}\right)$ has been the focus of a number of studies. ${ }^{13-22}$ The photodissociation at 248 or $235 \mathrm{~nm}$ proceeds via an excitation from the ground $S_{0}$ state to the first excited singlet state, $S_{1}$, that has an $\mathrm{n} \pi^{*} \mathrm{C}=\mathrm{O}$ electronic configuration in the Franck-Condon region. Thus, the initial force acts to stretch the $\mathrm{C}-\mathrm{O}$ bond. The $\mathrm{C}-\mathrm{Cl}$ bond cleavage occurs via a transition state on $\mathrm{S}_{1}$ formed from the avoided crossing between the $\mathrm{n}^{*}{ }_{\mathrm{C}=\mathrm{O}}$ and $\mathrm{n} \sigma^{*}{ }_{\mathrm{C}-\mathrm{Cl}}$ configuration states. ${ }^{13,22}$ Typically, asymmetric aliphatically substituted aldehydes and ketones dissociate via a Norrish type I process, ${ }^{14,16}$ with the assumption that the weaker of the two $\alpha$-bonds preferentially breaks. In contrast, the bond that breaks upon UV excitation of acetyl chloride depends on the relative barrier heights on the excited state potential energy surface.

\section{Previous Experimental Results}

The key experimental observable used to test the model is the measured velocity spectrum of the $\mathrm{CH}_{3} \mathrm{CO}$ radicals that are formed with too little vibrational energy to undergo further dissociation to $\mathrm{CH}_{3}+\mathrm{CO}$.

$$
\begin{aligned}
& \left.\mathrm{CH}_{3} \mathrm{COCl} \stackrel{h v}{\longrightarrow} \mathrm{CH}_{3} \mathrm{CO}+\mathrm{Cl}^{2}{ }^{2} \mathrm{P}_{J}\right) \quad J=\frac{1}{2}, \frac{3}{2} \\
& \mathrm{CH}_{3} \mathrm{CO} \rightarrow \mathrm{CH}_{3}+\mathrm{CO}
\end{aligned}
$$

Many experimental studies have investigated the dissociation dynamics and barrier for the dissociation of $\mathrm{CH}_{3} \mathrm{CO}^{8,13,15,17,20,23}$ Here we compare our model's predictions for the energy partitioning to the $\mathrm{CH}_{3} \mathrm{CO}$ radicals produced in the photodissociation of acetyl chloride to the experimental results in ref 13. Those velocity map imaging experiments determined the total recoil kinetic energy spectrum for the $\mathrm{C}-\mathrm{Cl}$ bond photofission of acetyl chloride at $235 \mathrm{~nm}$, producing both stable and unstable $\mathrm{CH}_{3} \mathrm{CO}$ radicals, and the velocity spectrum of the stable radicals to determine which radicals formed from $\mathrm{C}-\mathrm{Cl}$ bond fission were stable to subsequent dissociation. The details of this apparatus and the experimental results are described

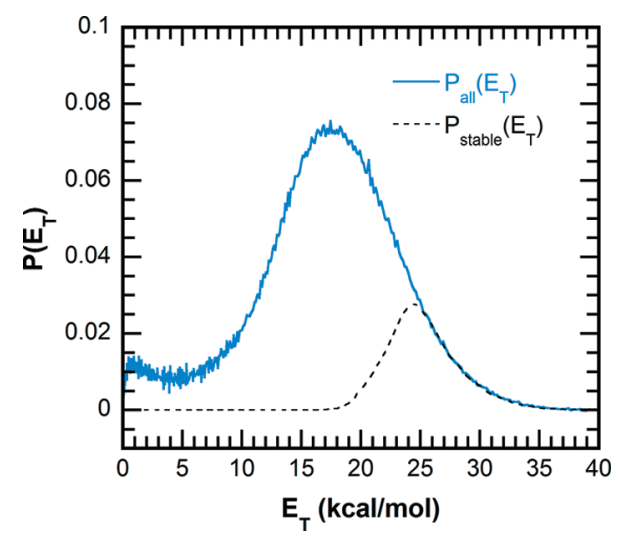

Figure 1. Center of mass recoil kinetic energy distributions of all $\mathrm{C}-\mathrm{Cl}$ bond fission events, $P_{\text {all }}\left(E_{\mathrm{T}}\right)$, in blue, and just those events that result in stable radicals, $P_{\text {stable }}\left(E_{\mathrm{T}}\right)$, in black dashed line. Adapted from ref 13.

elsewhere. ${ }^{1,13}$ In short, a supersonic molecular beam of acetyl chloride molecules was photodissociated at $235 \mathrm{~nm}$, and the $\mathrm{Cl}$ and $\mathrm{CH}_{3} \mathrm{CO}$ cofragments were photoionized and detected with velocity map imaging to measure the distributions of velocities, $P_{\mathrm{Cl}}(v)$ and $P_{\text {acetyl }}(v)$ respectively. The acetyl radicals were produced in a range of velocities corresponding to internal energies that span the barrier to further dissociation. Those acetyl radicals with low velocities have a significant amount of internal energy and will dissociate on a time scale much faster than the detection time scale (about $40 \mathrm{~ns}$ ) and thus will not be detected. Conservation of momentum of the cofragments allows the calculation of the distribution of total recoil kinetic energies of all dissociation events, $P_{\text {all }}\left(E_{\mathrm{T}}\right)$, from $P_{\mathrm{Cl}}(v)$, as well as the range of total recoil kinetic energies of the dissociation events resulting in stable acetyl radicals, $P_{\text {stable }}\left(E_{\mathrm{T}}\right)$, from $P_{\text {acetyl }}(v)$, shown in Figure 1. As expected, $P_{\text {all }}\left(E_{\mathrm{T}}\right)$ and $P_{\text {stable }}\left(E_{\mathrm{T}}\right)$ match at high translational energies (i.e., low internal energies) but begin to deviate around $25 \mathrm{kcal} / \mathrm{mol}$, and $P_{\text {stable }}\left(E_{\mathrm{T}}\right)$ is equal to zero at energies below $19 \mathrm{kcal} / \mathrm{mol}$. The energy at which the two distributions begin to diverge may be used to calculate an upper bound to the experimental barrier height for $\mathrm{CH}_{3} \mathrm{CO}$ dissociation. Our goal is to predict the distribution of recoil kinetic energies that resulted in $\mathrm{CH}_{3} \mathrm{CO}$ radicals stable to subsequent dissociation, the $P_{\text {stable }}\left(E_{\mathrm{T}}\right)$ distribution in the black dashed line in Figure 1. First, we review simple energy conservation arguments and the ab initio numbers used in the model.

At each measured recoil kinetic energy $E_{\mathrm{T}}$, one may use energy conservation to calculate the internal energy of the acetyl radical, $E_{\text {int }}$ (acetyl radical):

$$
\begin{array}{r}
E_{h v}+E_{\text {int }}(\text { acetyl chloride })=D_{0}(\mathrm{C}-\mathrm{Cl})+E_{\mathrm{T}}+ \\
\left.E_{\text {int }}\left(\mathrm{Cl}^{2} \mathrm{P}_{J}\right]\right)+E_{\text {int }}(\text { acetyl radical })
\end{array}
$$

Here $E_{h v}=121.5 \mathrm{kcal} / \mathrm{mol}$ is the energy of the photon that breaks the $\mathrm{C}-\mathrm{Cl}$ bond. $E_{\text {int }}$ (acetyl chloride) is the internal energy of the parent molecules. It has an average value of $1.1 \mathrm{kcal} /$ mol, but it will be demonstrated shortly that simply using an average value yields a poor prediction for the distribution of stable radicals. $D_{0}(\mathrm{C}-\mathrm{Cl})$ is the bond dissociation energy of the $\mathrm{C}-\mathrm{Cl}$ bond, experimentally determined as $83.62 \pm 0.15$ $\mathrm{kcal} / \mathrm{mol},{ }^{24,25}$ which is quite close to the theoretical value calculated at the UCCSD(T)/CBS//UCCSD(T)/aug-cc-pVTZ level of theory as $83.4 \mathrm{kcal} / \mathrm{mol} .{ }^{13}$ It is slightly higher than the G4//B3LYP/6-311++G(3df,2p) value of $82.57 \mathrm{kcal} / \mathrm{mol} .{ }^{26}$ 
$E_{\text {int }}\left(\mathrm{Cl}\left[{ }^{2} \mathrm{P}_{J}\right]\right)$ is either 0 or $2.52 \mathrm{kcal} / \mathrm{mol}$ depending on which spin-orbit state of chlorine is detected. $E_{\mathrm{T}}$ is derived from the velocity distributions measured by state-selectively detecting the $\mathrm{Cl}\left({ }^{2} \mathrm{P}_{1 / 2}\right)$ and $\mathrm{Cl}\left({ }^{2} \mathrm{P}_{3 / 2}\right)$ atoms and adding the two distributions. This calculation yields the total internal energy of the acetyl radicals, $E_{\text {int }}$ (acetyl radical), at each $E_{\mathrm{T}}$, but one must model the partitioning of this internal energy between rotational and vibrational energy to predict the distribution of radicals measured to be stable to subsequent dissociation. (Unless the moment of inertia of the radicals is very different in the molecular region and the transition state for dissociation to $\mathrm{CH}_{3}+\mathrm{CO}$, none of the energy partitioned to rotational energy can be used to surmount the dissociation barrier.) We describe our model below and compare its predictions with the experimental results.

\section{Development of the Model}

For each measured relative kinetic energy in the measured $P_{\text {all }}\left(E_{\mathrm{T}}\right)$ for $\mathrm{C}-\mathrm{Cl}$ bond fission, the model invokes conservation of angular momentum to predict the rotational angular momentum imparted to the radical. As the supersonic expansion of the parent molecules ${ }^{8}$ effectively cools molecular rotation, the initial angular momentum is taken as zero (this classical model does not include the photon's angular momentum, which typically couples to electronic angular momentum). Thus the total angular momentum of the fragments must also be zero following the dissociation.

$$
\vec{J}_{\text {total }}=\vec{J}_{\text {orb }}+\vec{J}_{\text {rot }}=0
$$

The classical orbital angular momentum of the fragments $\vec{J}_{\text {orb}}$, with magnitude $\left(\mu\left|v_{\text {rel }}\right| b\right)$, is equal in magnitude and opposite in direction to the rotational angular momentum $\vec{J}_{\text {rot }}$ of the acetyl radical product $(I \omega)$, where $\mu$ is the reduced mass of the acetyl $+\mathrm{Cl}$ system, $v_{\text {rel }}$ is the relative velocity between the departing cofragments, and $b$ is the impact parameter when the impulsive force dissociates the $\mathrm{C}-\mathrm{Cl}$ bond. $\mathrm{I}$ is the moment of inertia of the acetyl radical about its axis of rotation, and $\omega$ is the angular velocity of the acetyl radical. Our calculation assumes that the force acts impulsively along the $\mathrm{C}-\mathrm{Cl}$ bond, so we use the geometry of the $\mathrm{CH}_{3} \mathrm{CO}$ moiety in acetyl chloride to calculate I. Substitution of the definitions of rotational energy $\left(E_{\mathrm{rot}}=\right.$ $\left.(1 / 2) I \omega^{2}\right)$ and translational energy $\left(E_{\mathrm{T}}=(1 / 2) \mu v_{\text {rel }}^{2}\right)$ yields the required expression for the rotational energy imparted to the acetyl radical for each recoil kinetic energy $E_{\mathrm{T}}$ measured in the experiment:

$$
E_{\mathrm{rot}}=\frac{\mu b^{2}}{I} E_{\mathrm{T}}
$$

The $\left(\mu b^{2}\right) / I$ factor in eq 4 can be calculated by a series of vector calculations, given the Cartesian coordinates of the acetyl chloride geometry at which the impulsive force acts.

We note that the model prediction for the stable radical spectrum as implemented here does not account for the geometry difference between the acetyl moiety in acetyl chloride and the $\mathrm{CH}_{3} \mathrm{CO}$ radical nor for the change in rotational energy of the $\mathrm{CH}_{3} \mathrm{CO}$ radical as the $\mathrm{C}-\mathrm{C}$ bond stretches. If the moment of inertia increases, the classical rotational angular momentum stays constant but the energy partitioned to rotational energy decreases from that calculated here. Our impulsive model could allow for this correction to be made, as we also calculate the axis about which the radical is rotating.
To test the accuracy of the model in predicting the measured distribution of radical stable to subsequent dissociation, we substitute eq 4 into eq 2 and rearrange to get

$$
\begin{array}{r}
E_{\mathrm{vib}}(\text { radical })=E_{h v}+E_{\text {int }}(\text { acetyl chloride })-D_{0}(\mathrm{C}-\mathrm{Cl})- \\
E_{\mathrm{int}}\left(\mathrm{Cl}^{2}\left[\mathrm{P}_{J}\right]\right)-\left(1+\frac{\mu b^{2}}{I}\right) E_{\mathrm{T}}
\end{array}
$$

At each measured $E_{\mathrm{T}}$, if enough energy is partitioned to rotation the radical is left with vibrational energy lower than the calculated barrier for its subsequent dissociation. Thus the model's prediction for the distribution of stable radicals is sensitive to the impact parameter $b$ calculated from the geometry at which the impulsive force acts. We explore this in the following section.

\section{Model Predictions}

The simplest prediction assumes the impulsive force acts in the Franck-Condon region, so the ground state equilibrium geometry of acetyl chloride is taken as the geometry at which to calculate $\left(\mu b^{2}\right) / I$. Using the average internal energy of the precursor of $1.1 \mathrm{kcal} / \mathrm{mol}$ and the experimental $\mathrm{C}-\mathrm{Cl}$ bond dissociation energy of $83.62 \mathrm{kcal} / \mathrm{mol}$, we can calculate the simplest estimate for the measured distribution of stable radicals, $P_{\text {stable }}\left(E_{\mathrm{T}}\right)$, from the measured distribution of recoil kinetic energies. (We do this by subtracting the contribution from any radicals with $E_{\mathrm{vib}}$ greater than the theoretically calculated $\operatorname{CCSD}(\mathrm{T})$ barrier height, $16.77 \mathrm{kcal} / \mathrm{mol},{ }^{8}$ from the total distribution, $P_{\mathrm{all}}\left(E_{\mathrm{T}}\right)$, as these will dissociate and not be detected.) The distribution of vibrational energies predicted from this simplest model is shown in the upper panel of Figure 2, and the model's prediction for $P_{\text {stable }}\left(E_{\mathrm{T}}\right)$ is shown in red in the lower frame superimposed on the experimentally measured distribution. The predicted distribution of stable radicals is bimodal, with two sharp $E_{\mathrm{T}}$ onsets for the dissociation of $\mathrm{CH}_{3} \mathrm{CO}$ radicals, one for radicals formed in conjunction with $\mathrm{Cl}\left({ }^{2} \mathrm{P}_{1 / 2}\right)$ and one for those formed in conjunction with $\mathrm{Cl}\left({ }^{2} \mathrm{P}_{3 / 2}\right)$. Clearly this simple prediction does adequately match the experimentally measured spectrum of stable radicals.

Our improved model ${ }^{1}$ accounts for the change in the $\left(\mu b^{2}\right) / I$ factor over the quantum vibrational wave function of the dissociating molecule (for example, due to the range of impact parameters from zero point motion in a bend or pyramidal vibration). Additionally, the model explicitly includes the entire thermal distribution of vibrational energies of the ground state acetyl chloride molecules, calculated from a direct count of states before they absorb a photon, which broadens the predicted $P_{\text {stable }}\left(E_{\mathrm{T}}\right)$ considerably. We include only the normal mode of vibration that results in the most significant change in the $\left(\mu b^{2}\right) / I$ factor to estimate the first correction. Using the equilibrium geometry, displacement vectors, force constants, and scaled ${ }^{27}$ frequencies calculated at B3LYP/6-311++G(d,p) level of theory, ${ }^{28}$ the $\left(\mu b^{2}\right) / I$ factor is calculated for 40 geometries, some extending beyond the classical turning points. Figure 3 shows the resulting prediction for the distribution of acetyl radicals stable to subsequent dissociation, $P_{\text {stable }}\left(E_{\mathrm{T}}\right)$, compared with the measured spectrum. The prediction is much improved and more adequately predicts the full distribution of stable radicals. It is encouraging that this prediction uses no variable parameters. We use the experimental value for the $\mathrm{C}-\mathrm{Cl}$ bond dissociation energy and the $\operatorname{CCSD}(\mathrm{T})$ ab initio value for the barrier for the subsequent dissociation for $\mathrm{CH}_{3} \mathrm{CO}$ radicals. Nevertheless, assuming the impulsive force acts at the equilibrium geometry 


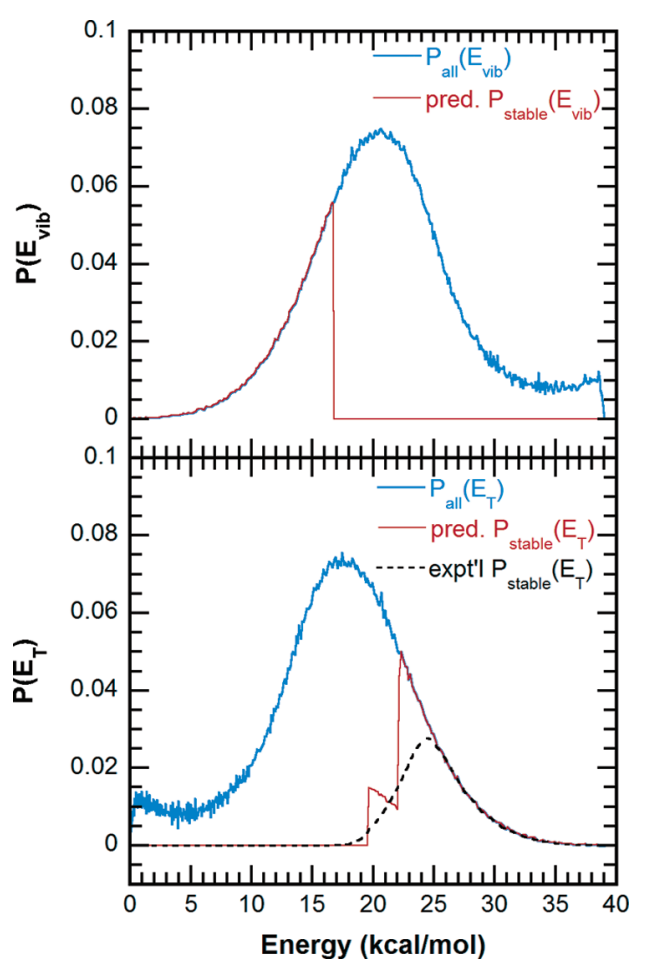

Figure 2. Predictions from the simplest model (using a single impact parameter and $\left\langle E_{\text {int }}\right.$ (acetyl chloride $\left.)\right\rangle=1.1 \mathrm{kcal} / \mathrm{mol}$ ). In the upper frame, the distribution of vibrational energies of all radicals is shown in blue. The calculated barrier height of $16.77 \mathrm{kcal} / \mathrm{mol}$ is used to calculate the distribution of vibrational energies of stable radicals, shown in red. This distribution is converted to a $P_{\text {stable }}\left(E_{\mathrm{T}}\right)$ shown in red in the lower frame on top of the experimental $P_{\text {stable }}\left(E_{\mathrm{T}}\right)$ shown in black dashed line. The bimodal nature of the predicted $P_{\text {stable }}\left(E_{\mathrm{T}}\right)$ comes from the acetyl radicals formed in conjunction with $\mathrm{Cl}\left({ }^{2} \mathrm{P}_{1 / 2}\right)$ and $\mathrm{C}\left({ }^{2} \mathrm{P}_{3 / 2}\right)$, which accounts for an available energy difference of $2.52 \mathrm{kcal} / \mathrm{mol}$.

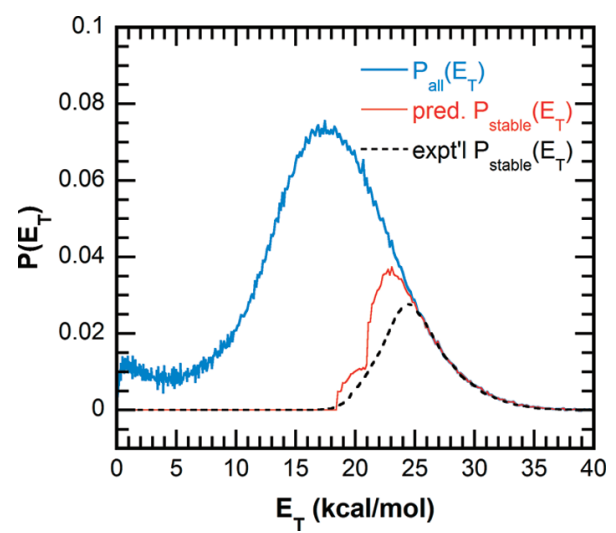

Figure 3. Predicted $P_{\text {stable }}\left(E_{\mathrm{T}}\right)$ using the ground state geometry of acetyl chloride and considering a range of geometries as the molecule vibrates along the $v_{3}$ normal mode $\left(338 \mathrm{~cm}^{-1}\right)$ shown in red, superimposed on the experimentally determined spectrum in black dashed line.

of acetyl chloride is not physically reasonable given the nature of the excited state, and we note that the predicted distribution retains a bimodal character that is not reproduced in the experimental results.

Because the excited state is not repulsive in the Franck-Condon region, it unlikely that the impulsive force acts at the geometry on $\mathrm{S}_{1}$ corresponding to the ground state equilibrium geometry. We thus consider the possibility that the impulsive force along the $\mathrm{C}-\mathrm{Cl}$ bond that generates rotational energy in the acetyl radical acts when the acetyl chloride is at the transition state geometry on $\mathrm{S}_{1}$ calculated by Chen and Fang. ${ }^{22}$ Researchers

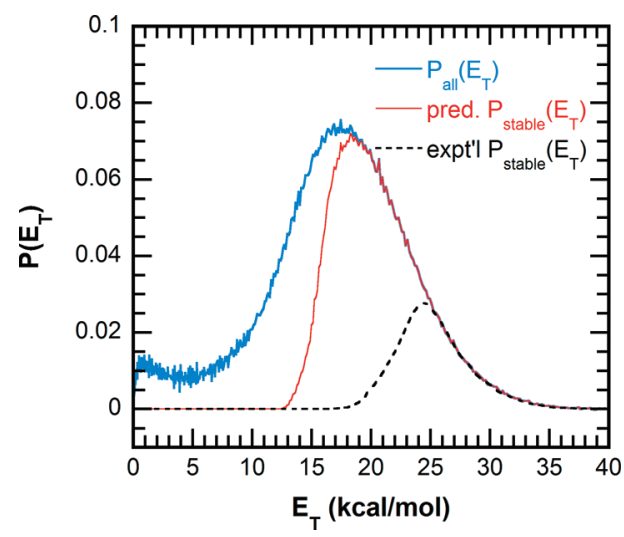

Figure 4. Predicted $P_{\text {stable }}\left(E_{\mathrm{T}}\right)$ using the transition state geometry of acetyl chloride and considering a range of geometries as the molecule vibrates along the $v_{5}$ normal mode $\left(444 \mathrm{~cm}^{-1}\right)$ shown in red, superimposed on the experimentally determined spectrum, shown in black dashed line.

using North's modified impulsive model for reactions with an exit barrier ${ }^{8}$ typically use the transition state ${ }^{29,30}$ in conjunction with impulsive models to predict the nonstatistical energy partitioning to translational and rotational degrees of freedom, so we now attempt to use this geometry for our impulsive model. This transition state geometry has an elongated $\mathrm{C}-\mathrm{Cl}$ bond, and the bonding about the $\alpha$-carbon has become more pyramidal, resulting in a much larger impact parameter and much more partitioning of energy to the rotation of the acetyl radical. Surprisingly, the resulting $P_{\text {stable }}\left(E_{\mathrm{T}}\right)$, shown in red in Figure 4, predicts far too much energy is partitioned into rotation for it to agree with the experimental data. Evidently, the commonly accepted notion that nonstatistical energy partitioning results from forces at or beyond the transition state needs to be reevaluated.

The above two comparisons suggest that the geometry at which an impulsive force acts to break the $\mathrm{C}-\mathrm{Cl}$ bond is somewhere in between the Franck-Condon region and the transition state, where the $\alpha$-carbon has started to become slightly pyramidal but does not yet have the large impact parameter calculated at the transition state. To make a choice for this geometry for use in our model, we consider the trajectory calculations on $S_{1}$ published by Chen and Fang (ref 22) and reproduced in Figure 5. Note that although the $\mathrm{C}-\mathrm{O}$ bond stretches to a length of $1.673 \AA$ in the first 50 fs of the excitation before returning to oscillate about its equilibrium value, the $\mathrm{C}-\mathrm{Cl}$ bond length increases nearly monotonically after an initial delay of $25 \mathrm{fs}$. In the first $25 \mathrm{fs}$ or so, the $\mathrm{C}-\mathrm{Cl}$ bond remains close to its equilibrium geometry. If one had to approximate the $\mathrm{C}-\mathrm{Cl}$ bond fission as occurring due to an impulsive force, one should identify in this trajectory the time at which $\mathrm{d} R(\mathrm{C}-\mathrm{Cl}) / \mathrm{d} t$ becomes large and positive and then stays nearly constant. Thus, in our model we use the geometry at the outer turning point of the $\mathrm{C}-\mathrm{O}$ stretch as the geometry at which an impulsive dissociation generates relative kinetic energy in the $\mathrm{C}-\mathrm{Cl}$ bond fission and rotation in the acetyl radical. That geometry is given in Table 1, along with the resulting impact parameter and axis of rotation and moment of inertia for the evolving acetyl radical. The impact parameter for the $\mathrm{C}-\mathrm{Cl}$ bond fission at this geometry is significantly smaller than that at the transition state for $\mathrm{C}-\mathrm{Cl}$ bond fission, so our model predicts a smaller partitioning of energy to rotation at each measured $E_{\mathrm{T}}$. The predicted $P_{\text {stable }}\left(E_{\mathrm{T}}\right)$ values shown in the upper and lower frames of Figure 6 are derived using the geometry calculated at the CAS $(10,8) / c c-p V D Z$ level of theory in 

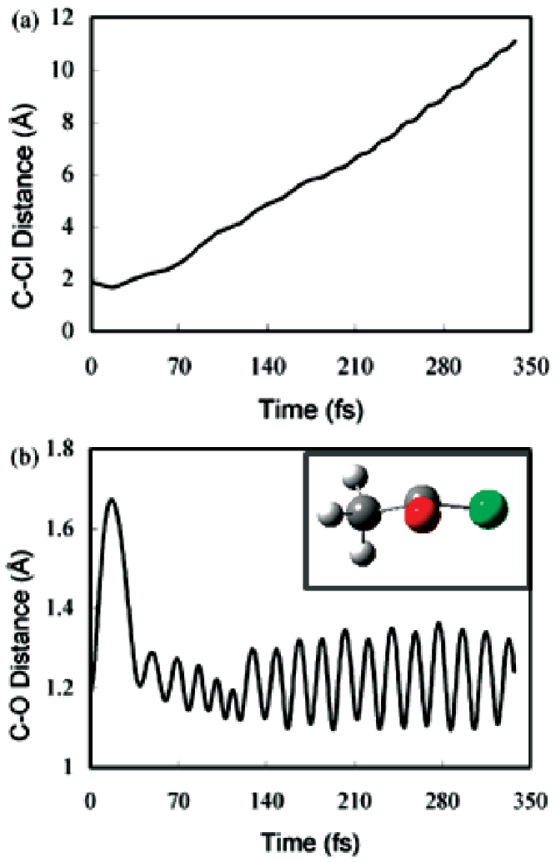

Figure 5. Results of the trajectory calculations beginning at the Franck-Condon geometry on $\mathrm{S}_{1}$, adapted from ref 22 . The $\mathrm{C}-\mathrm{Cl}$ bond length (frame a) and the $\mathrm{C}-\mathrm{O}$ bond length (frame b) are shown as a function of time. The inset in frame $\mathrm{b}$ shows the slightly pyramidal geometry of acetyl chloride at the turning point of the trajectory at which the $\mathrm{C}-\mathrm{O}$ bond length is 1.673 .

TABLE 1: Cartesian Coordinates of the Acetyl Chloride Molecule at the Outer Turning Point of the $\mathrm{C}-\mathrm{O}$ Stretch Trajectory $^{a}$

\begin{tabular}{lccc}
\hline & $X$ & $Y$ & $Z$ \\
\hline $\mathrm{C}$ & -0.34786 & 0.045554 & -0.1904 \\
$\mathrm{O}$ & -0.847852 & 1.625079 & 0.042194 \\
$\mathrm{Cl}$ & 1.316225 & -0.170072 & 0.020705 \\
$\mathrm{C}$ & -1.409924 & -1.069059 & 0.03643 \\
$\mathrm{H}$ & -1.375849 & -1.801522 & -0.77449 \\
$\mathrm{H}$ & -1.291133 & -1.612652 & 0.981776 \\
$\mathrm{H}$ & -2.379414 & -0.554199 & 0.02701
\end{tabular}

${ }^{a}$ The unscaled vibrational frequencies, force constants, and displacement vectors were calculated from this geometry at the CAS(10,8)/cc-pVDZ level of theory. At this geometry, the impact parameter is $0.2864 \AA$ and the moment of inertia is 16.4922 $\mathrm{amu} \cdot \AA^{2}$.

Table 1 and averaged over the third normal mode of vibration (312 $\mathrm{cm}^{-1}$ unscaled). The bimodal nature of the previous prediction has vanished because the impulsive force acting at this geometry imparts a wider range of $\left(\mu b^{2}\right) / I$ factors, broadening the predicted spectrum. The upper frame uses the experimental value of $D_{0}(\mathrm{C}-\mathrm{Cl})$ in the energy conservation relation given in eq 2 and allows all radicals to dissociate if they have a vibrational energy higher than the ab initio barrier of 16.77 $\mathrm{kcal} / \mathrm{mol}$. While there are no direct experimental measurements of this barrier, the zero-point corrected $\mathrm{C}-\mathrm{C}$ bond dissociation energy for the dissociation of $\mathrm{CH}_{3} \mathrm{CO}$ to $\mathrm{CH}_{3}+\mathrm{CO}$ has been measured as $9.31 \pm 0.15 \mathrm{kcal} / \mathrm{mol},{ }^{24,25}$ quite close to the CCSD(T) value of $9.45 \mathrm{kcal} / \mathrm{mol}{ }^{31}$ The lower frame gives the result when one iteratively adjusts the sum of $D_{0}$ and the barrier down by $2.77 \mathrm{kcal} / \mathrm{mol}$, so $D_{0}(\mathrm{C}-\mathrm{Cl})+E_{\text {barrier }}$ is set as 97.62 $\mathrm{kcal} / \mathrm{mol}$. The model prediction in the lower frame gives an excellent fit to the experimentally measured spectrum of stable radicals. Figure 7 gives the corresponding vibrational energy distribution of all the nascent radicals, both stable and unstable,

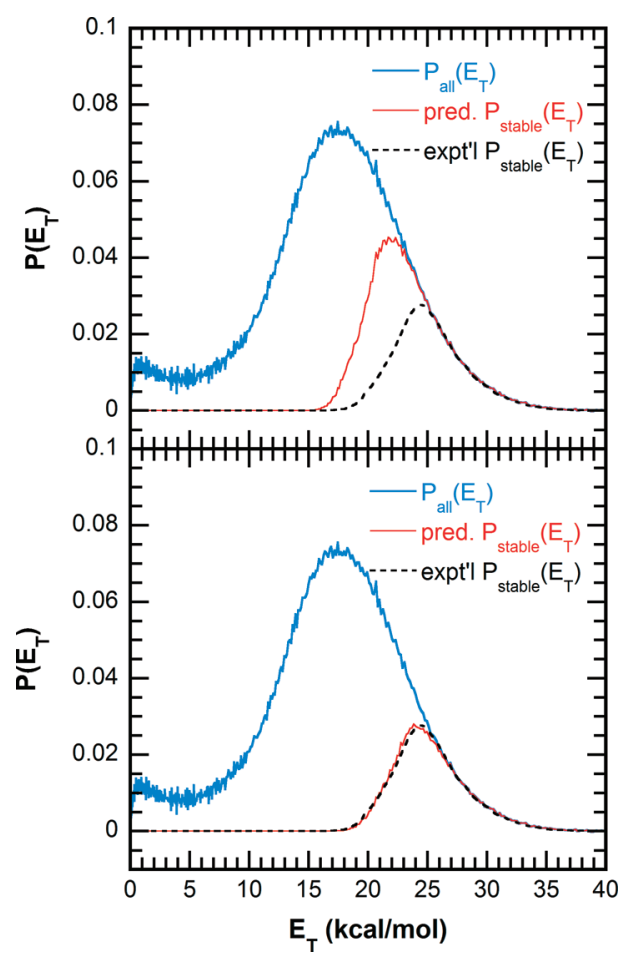

Figure 6. Predicted $P_{\text {stable }}\left(E_{\mathrm{T}}\right)$ using the geometry, from the trajectory results in Figure 5, at the outer turning point of the $\mathrm{C}-\mathrm{O}$ stretch and considering a range of geometries accessed during the zero-point motion of the $v_{3}$ normal mode $\left(312 \mathrm{~cm}^{-1}\right.$ unscaled). The upper frame shows the predicted spectrum using the values for $D_{0}$ and the $\operatorname{CCSD}(\mathrm{T})$ calculated barrier height, 83.62 and $16.77 \mathrm{kcal} / \mathrm{mol}$, respectively. ${ }^{7}$ The lower frame shows the predicted spectrum if one reduces the sum of $D_{0}+$ barrier height by $2.77 \mathrm{kcal} / \mathrm{mol}$ in the model input.

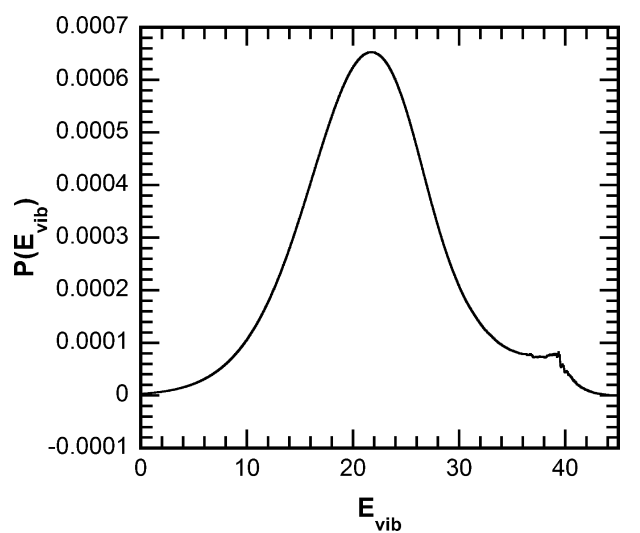

Figure 7. Predicted vibrational energy distribution of all the nascent radicals, both stable and unstable, assuming that the value of $D_{0}(\mathrm{C}-\mathrm{Cl})$ in acetyl chloride is $80.85 \mathrm{kcal} / \mathrm{mol}$ and convoluting over the range of geometries accessed during the zero-point motion of the $v_{3}$ normal mode (312 $\mathrm{cm}^{-1}$ unscaled).

assuming the value of $D_{0}(\mathrm{C}-\mathrm{Cl})$ in acetyl chloride with the same model parameters that gave the fit in the lower frame of Figure 6. (It is interesting to note that the derived $P\left(E_{\mathrm{vib}}\right)$ spectrum is relatively insensitive to the model parameters as long as we choose a set of parameters that gives a good fit to the $P_{\text {stable }}\left(E_{\mathrm{T}}\right)$ spectrum. For example, the $P\left(E_{\mathrm{vib}}\right)$ in Figure 7 is nearly identical to the $P\left(E_{\mathrm{vib}}\right)$ derived from an impulsive model at the equilibrium geometry if one lowers $D_{0}$ by $1.32 \mathrm{kcal} / \mathrm{mol}$ to improve the fit shown in Figure 3.) We note that the adjustment required to get a good fit, decreasing the sum $D_{0}(\mathrm{C}-\mathrm{Cl})+$ acetyl dissociation barrier down by $2.77 \mathrm{kcal} / \mathrm{mol}$, is outside the range of uncertainty in the experimental $D_{0}$ and 
$\mathrm{ab}$ initio barrier prediction. A model prediction using a distribution of geometries slightly less pyramidal than the one used for demonstration purposes here could give a good fit to the data without the large adjustment, but we would need an ensemble of classical trajectories on $\mathrm{S}_{1}$ to make this prediction. Even at this simple level, the modeling here has conclusively shown that assuming an impulsive force acting from the transition state for $\mathrm{C}-\mathrm{Cl}$ bond fission on $\mathrm{S}_{1}$ gives a very poor prediction for the spectrum of stable radicals; the $\mathrm{C}-\mathrm{Cl}$ bond experiences an impulsive force in a region of the $\mathrm{S}_{1}$ excited state that is much less pyramidal.

\section{Discussion and Summary}

For studies of the unimolecular and bimolecular reactions of radicals in the gas phase, it is important to have a rough estimate of the distribution of vibrational energy in the nascent radicals. This work tests the extension of a recent model for obtaining the rotational and vibrational energy distribution of radicals produced from the photodissociation of a halogenated precursor for the special case that the dissociative excited state of the precursor is not repulsive in the Franck-Condon region. Using only the measured distribution of recoil kinetic energies in the $\mathrm{C}$-halogen bond fission, we model the partitioning of energy to rotation of the radical impulsively and use this to predict the vibrational energy distribution of the nascent radicals and the portion of the distribution of $\mathrm{C}$-halogen bond fission recoil kinetic energy distribution that produced stable radicals. Our analysis shows that the choice of geometry for the impulsive dissociation is critical. Though prior studies using impulsive models commonly assume that one should use the geometry of the transition state for $\mathrm{C}$-halogen bond fission when one predicts the amount of energy partitioned impulsively to product rotation, we show here that this assumption is a poor one. For acetyl chloride dissociation, it predicts too much rotational energy in the nascent radicals. We thus find that for most of the dissociative trajectories the impulsive $\mathrm{C}-\mathrm{Cl}$ bond fission begins at a geometry that is not as pyramidal as that represented by the transition state for $\mathrm{C}-\mathrm{Cl}$ bond fission on $\mathrm{S}_{1}$. The work demonstrates that one should not assume that impulsive forces acting near the transition state are important for the actual energy partitioning. Rather, it is preferable to examine calculated trajectories on the excited state to arrive at a reasonable geometry to use for such impulsive models. When one does that, the extended model presented here, which accounts for the distribution of internal energies in the halogenated precursor as well as the variation in impact parameter due to vibrational motion in the precursor, gives a quite good fit to the measured distribution of stable radicals. One can understand the results in the acetyl chloride system by considering the multidimensional nature of the global potential energy surface for $S_{1}$ and the associated trajectories on it. The results suggest that the majority of the dissociative trajectories for $\mathrm{C}-\mathrm{Cl}$ bond fission sample the avoided crossing between the $\mathrm{n} \pi^{*} \mathrm{C}=\mathrm{O}$ and $\mathrm{n} \sigma^{*}{ }_{\mathrm{C}-\mathrm{Cl}}$ configuration states not at the calculated transition state, but rather at a geometry that is not as pyramidal.

Acknowledgment. This work was supported by the Chemical Sciences, Geosciences and Biosciences Division, Office of Basic Energy Sciences, Office of Science, U.S. Department of Energy, under Grant No. DE-FG02-92ER14305 (Butler).

\section{References and Notes}

(1) Ratliff, B. J.; Womack, C. C.; Tang, X. N.; Landau, W. L.; Butler, L. J.; Szpunar, D. E. J. Phys. Chem. A 2010, 114, 4934.

(2) Levine, R. D.; Bernstein, R. B. Acc. Chem. Res. 1974, 7, 393.

(3) Band, Y. B.; Freed, K. F. J. Chem. Phys. 1975, 63, 3382.

(4) Mitchell, R. C.; Simons, J. P. Discuss. Faraday Soc. 1967, 44, 208. 132.

(5) Riley, S. J.; Wilson, K. R. Faraday Discuss. Chem. Soc. 1972, 53,

(6) Busch, G. E.; Wilson, K. R. J. Chem. Phys. 1972, 56, 3626.

(7) Holdy, K. E.; Klotz, L. C.; Wilson, K. R. J. Chem. Phys. 1970, 52, 4588

(8) North, S. W.; Blank, D. A.; Gezelter, J. D.; Longfellow, C. A.; Lee, Y. T. J. Chem. Phys. 1995, 102, 4447.

(9) Krisch, M. J.; McCunn, L. R.; Takematsu, K.; Butler, L. J.; Blase, F. R.; Shu, J. J. Phys. Chem. A 2004, 108, 1650.

(10) Szpunar, D E. Ph.D. Thesis, Appendix C, The University of Chicago, Chicago, IL, 2003.

(11) Hintsa, E. J.; Zhao, X.; Lee, Y. T. J. Chem. Phys. 1990, 92, 2280.

(12) Sapers, S. P.; Hess, W. P. J. Chem. Phys. 1992, 97, 3126.

(13) Tang, X.; Ratliff, B. J.; FitzPatrick, B. L.; Butler, L. J. J. Phys. Chem. B 2008, 112, 16050.

(14) Person, M. D.; Kash, P. W.; Butler, L. J. J. Phys. Chem. 1992, 96, 2021.

(15) Deshmukh, S.; Myers, J. D.; Xantheas, S. S.; Hess, W. P. J. Phys. Chem. 1994, 98, 12535.

(16) Martin, X.; Moreno, M.; Lluch, J. M. J. Phys. Chem. 1993, 97, 12186.

(17) Shibata, T.; Li, H.; Katayanagi, H.; Suzuki, T. J. Phys. Chem. A 1998, 102, 3643.

(18) Sumathi, R.; Chandra, A. K. J. Chem. Phys. 1993, 99, 6531

(19) Deshmukh, S.; Hess, W. P. J. Chem. Phys. 1994, 100, 6429.

(20) North, S.; Blank, D. A.; Lee, Y. T. Chem. Phys. Lett. 1994, 224, 38.

(21) Shibata, T.; Suzuki, T. Chem. Phys. Lett. 1996, 262, 115.

(22) Chen, S.-L.; Fang, W.-H. J. Phys. Chem. A 2007, 111, 9355.

(23) Watkins, K. W.; Word, W. W. Int. J. Chem. Kinet. 1974, 6, 855.

(24) (a) Ruscic, B.; Pinzon, R. E.; Morton, M. L.; von Laszewski, G.; Bittner, S.; Nijsure, S. G.; Amin, K. A.; Minkoff, M.; Wagner, A. F. $J$. Phys. Chem. A 2004, 108, 9979-9997. (b) Ruscic, B. Private communication of unpublished interim results from Active Thermochemical Tables (ATcT) ver. 1.37 and the Core (Argonne) Thermochemical Network ver. 1.110.

(25) The revised experimental values given in ver. 1.37 of the ATcT include a new experimental determination of the heat of formation of acetyl radicals. Shuman, N. S.; Stevens, W. R.; Baer, T. Int. J. Mass. Spectrom. 2010, 294, 88 .

(26) Frisch, M. J., Trucks, G. W., Schegel, H. B. Gaussian 09, revision A.02; Gaussian, Inc.: Wallingford, CT, 2009.

(27) Scott, A. P.; Radom, L. J. Phys. Chem. 1996, 100, 16502.

(28) Frisch, M. J., Trucks, G. W., Schegel, H. B. Gaussian 03, revision E.01; Gaussian, Inc.: Wallingford, CT, 2004.

(29) Chang, A. H. H.; Hwang, D. W.; Yang, X.-M.; Mebel, A. M.; Lin, S. H.; Lee, Y. T. J. Chem. Phys. 1999, 110, 10810.

(30) SenGupta, S.; Upadhyaya, H. P.; Kumar, A.; Naik, P. D.; Bajaj, P. J. Chem. Phys. 2006, 124, 024305.

(31) FitzPatrick, B. L. Private communication, 2010. 